\title{
Urinary TIMP2.IGFBP7 for the prediction of platinum-induced acute renal injury
}

This article was published in the following Dove Press journal: International Journal of Nephrology and Renovascular Disease 28 June 2017

Number of times this article has been viewed

\author{
Moritz Schanz' \\ Anette Hoferer ${ }^{2}$ \\ Jing $\mathrm{Shi}^{3}$ \\ Mark Dominik Alscher' \\ Martin Kimmel' \\ 'Division of General Internal Medicine \\ and Nephrology, Department of \\ Internal Medicine, ${ }^{2}$ Division of \\ Oncology, Department of Internal \\ Medicine, Robert-Bosch Hospital, \\ Stuttgart, Germany; ${ }^{3}$ Walker \\ Bioscience, Carlsbad, CA, USA
}

Correspondence: Martin Kimmel Division of General Internal Medicine and Nephrology, Department of Internal Medicine, Robert-Bosch Hospital, Auerbachstrasse 1 10, 70376 Stuttgart, Germany

$\mathrm{Tel}+4971181013496$

Fax +4971181013792

Email martin.kimmel@rbk.de
Introduction: Platinum-based chemotherapy $(\mathrm{PBC})$ is a potent antineoplastic treatment, but cisplatin nephrotoxicity is often the limiting factor. Identifying the patients who are at risk for developing platinum-induced renal injury is an important issue. We tested urinary TIMP2-IGFBP7, a new US Food and Drug Administration (FDA)-cleared test to assess the risk of acute kidney injury (AKI), in a cohort of patients with malignant neoplastic disease receiving PBC.

Patients and methods: A total of 58 patients with malignant neoplastic disease were enrolled in this study, of whom 32 patients had both urine samples and subsequent serum creatinine values available for detecting AKI within 72 hours. Urine samples were collected within 6 hours prior to $\mathrm{PBC}$ application and within 12 hours after the end of chemotherapy administration. We examined the predictive ability of TIMP2-IGFBP7 for the development of AKI as defined by KDIGO (Kidney Disease: Improving Global Outcomes) criteria within 72 hours after the administration of chemotherapy. Operating characteristics were determined for the previously validated TIMP2·IGFBP7 cutoff of $0.3(\mathrm{ng} / \mathrm{mL})^{2} / 1000$.

Results: Four (12.5\%) patients developed AKI within 72 hours. Primary disease was lymphoma in 13 patients $(40.6 \%)$ and solid tumors in 19 patients (59.4\%). Eight patients $(25.0 \%)$ received carboplatin and 24 (75.0\%) cisplatin. TIMP2-IGFBP7 after PBC administration discriminated for the risk of AKI with an area under the receiver operating characteristic curve (AUC; 95\% confidence interval) of $0.92(0.80-1.00)$. At the cutoff of 0.3 for TIMP2-IGFBP7, sensitivity was $50 \%$, specificity was $87 \%$, negative predictive value was $95 \%$ and positive predictive value was $25 \%$ for the prediction of AKI within 72 hours.

Conclusion: Urinary TIMP2-IGFBP7 measured in specimens gathered after PBC may be a useful tool to early identify patients who are at risk for developing platinum-induced AKI.

Keywords: acute kidney injury, biomarker, cisplatin, nephrotoxicity, insulin like-growth factor binding protein-7, tissue inhibitor of metalloproteinases-2

\section{Introduction}

Cisplatin and platinum-based chemotherapy (PBC) are broadly used potent antineoplastic treatments. The cytotoxic substance, cisplatin, was first discovered in Escherichia coli as an anti-infective agent, and its antitumor activity was reported several years later. ${ }^{1,2}$ PBC is widely applied to treat oncologic diseases, such as lymphoma, testicular, ovarian, cervical and head- and neck non-small cell lung cancers, partially in combination with radiation therapy. ${ }^{3,4}$

As with most antitumor therapies, the major reason for PBC discontinuation is the noxious side effects. These side effects are well studied in cisplatin toxicity: the most severe, dose-limiting side effect is nephrotoxicity, which was reinforced by reports of renal failure after accidental overdose of cisplatin. ${ }^{5}$ Approximately one-third of the 
patients receiving cisplatin chemotherapy develop AKI at doses of $50-100 \mathrm{mg} / \mathrm{m}^{2}{ }^{6,7} \mathrm{AKI}$ is known to increase mortality and complicate hospital stay. ${ }^{8,9}$ Cisplatin nephrotoxicity is mostly mediated through inflammation, ${ }^{10,11}$ vasoconstriction $^{12,13}$ and direct cytotoxicity through reactive oxygen species. ${ }^{14}$ Renal proximal tubules show vulnerability to cisplatin toxicity that leads to a decline in GFR. ${ }^{15,16}$

To alleviate the nephrotoxic effects, prevention strategies such as hydration were established which allow the application of higher cisplatin doses. ${ }^{17,18}$ Development of carboplatin and other platinum-containing compounds led to less severe nephrotoxicity but did not eliminate nephrotoxic side effects. ${ }^{19,20}$ Despite hydration and new developments, nephrotoxicity remains a major reason for the discontinuation of therapy and complication of antitumor therapy. ${ }^{21}$

Over the past several years, new potential biomarkers for the early detection of AKI have been identified. ${ }^{22}$ TIMP2 and IGFBP7 are the most promising markers. Many studies have shown that the product of the concentration of these proteins TIMP2-IGFBP7 in urine (Astute Medical, San Diego, CA, USA) is an early indicator of risk for AKI.$^{23-28}$ As the first available AKI biomarker test in the USA, urinary TIMP2 $\cdot$ IGFBP7 was cleared in 2014 by the US FDA. ${ }^{29}$

In this study, we tested the hypothesis that urinary TIMP2-IGFBP7 collected directly prior and after PBC administration can predict the development of AKI. A rapid biomarker test, such as urinary TIMP2-IGFBP7, that can identify the patients who are at risk for developing PBCinduced AKI could lead to a more effective application of these antitumor agents or a further development of prevention strategies.

\section{Patients and methods}

This study was conducted at the Department of Internal Medicine at Robert-Bosch Hospital, Stuttgart (Germany), after obtaining approval from the ethics board of the University of Tuebingen (Germany). Urinary biomarkers were obtained in clinical routine, and the analysis was conducted retrospectively. Informed consent was not obtained due to the retrospective study design; patient confidentiality was maintained, and the collected data were anonymized without identifying information. Inclusion criteria were as follows: $\mathrm{sCr}$ values within normal range $(0.5-1.2 \mathrm{mg} / \mathrm{dL})$ at chemotherapy initiation, known malignant neoplastic disease, therapy with cisplatin or carboplatin and available follow-up $\mathrm{sCr}$ values. Exclusion criteria were as follows: already existing AKI or kidney impairment at the start of chemotherapy, no malignant neoplastic disease or no cisplatin/carboplatin chemotherapy. In this study, 26 patients were excluded due to missing subsequent $\mathrm{sCr}$ values (Figure 1). Six patients with baseline urinary TIMP2-IGFBP7 had missing urinary TIMP2·IGFBP7 samples after PBC administration. To identify eligible patients, the program for the preparation of cytotoxics was screened for $\mathrm{PBC}$ regimens (cisplatin and carboplatin). Urinary TIMP2-IGFBP7 was collected within 6 hours prior to PBC application and within 12 hours after the end of chemotherapy administration as a routine measurement. We examined the predictive ability of TIMP $2 \cdot$ IGFBP 7 for the development of AKI within 72 hours after the administration of chemotherapy. AKI was defined as at least stage 1 by $\mathrm{sCr}$ criteria of the KDIGO guideline. ${ }^{30}$ eGFR was calculated using the CKD-EPI equation. ${ }^{31}$ Test characteristics (sensitivity, specificity, NPV, PPV) were determined for the previously validated TIMP2-IGFBP7 cutoff of $0.3 .^{23}$

\section{Biomarker assays}

$\mathrm{SCr}$ and urinary TIMP2-IGFBP7 were measured at RobertBosch Hospital. Urinary TIMP2 and IGFBP7 concentrations were measured using the NephroCheck ${ }^{\mathrm{TM}}$ test and Astute140 Meter (Astute Medical, San Diego, CA, USA). TIMP2·IGFBP7 levels were reported in units of $(\mathrm{ng} / \mathrm{mL})^{2} / 1000$.

\section{Statistical analysis}

Predictive performance was assessed using ROC curve analysis, including the determination of the operating characteristics at the previously validated cutoff of $0.3 .95 \%$ CIs for AUCs were calculated with the DeLong method. 95\% CIs for operating characteristics were constructed with the Clopper-Pearson method. For comparisons of baseline

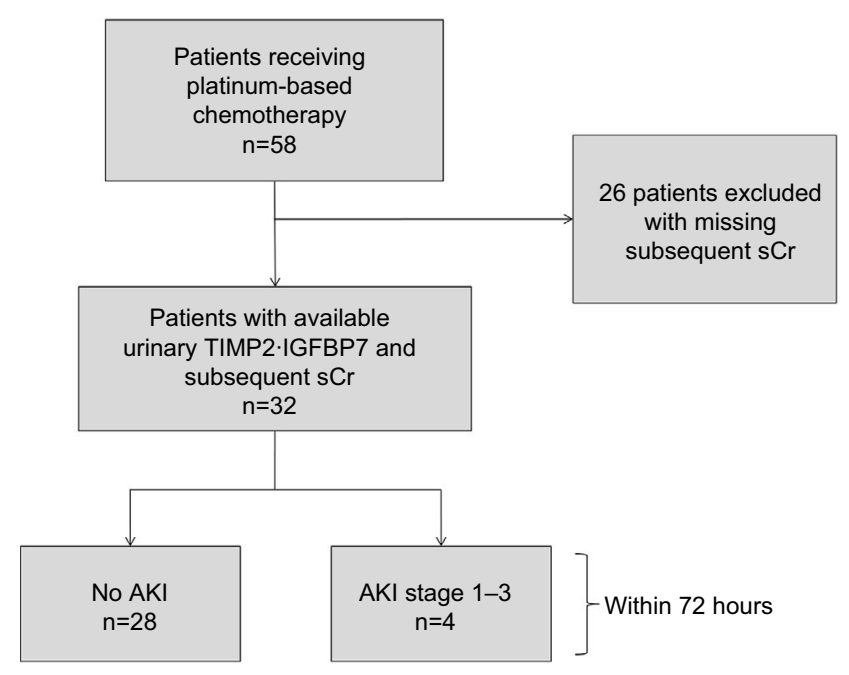

Figure I Study flow diagram

Abbreviations: $\mathrm{AKI}$, acute kidney injury; $\mathrm{s} \mathrm{Cr}$, serum creatinine. 
characteristics in end point negative and positive patients, categorical variables were analyzed using the Fisher's exact or chi-square test and continuous variables using the unpaired $t$-test and Wilcoxon rank-sum test, respectively, for normally and non-normally distributed variables. For comparison of paired samples, Wilcoxon matched-pairs signed-rank test was used. For all analyses, two-sided $p$-values of $<0.05$ and one-sided $p$-values of $<0.025$ were considered statistically significant. Statistical analysis was performed using Prism (GraphPad Software, Inc., La Jolla, CA, USA).

\section{Results}

\section{Baseline characteristics}

Four (12.5\%) patients developed AKI stage 1 within 72 hours. In each patient, urinary TIMP2-IGFBP7 samples after PBC administration were gathered before an AKI-defining rise in $\mathrm{sCr}$ was evident. No subject developed AKI stage 2 or 3. $\mathrm{SCr}$ increase was significantly greater $(p<0.0001)$ in the AKI group (median: $0.5 \mathrm{mg} / \mathrm{dL}$; IQR: $0.3-0.8$ ) than in the
non-AKI group (median: $0.0 \mathrm{mg} / \mathrm{dL}$; IQR: 0.0-0.1). eGFR decrease was significantly $(p=0.0004)$ greater in patients with AKI (median: $-28.3 \mathrm{~mL} / \mathrm{min} / 1.73 \mathrm{~m}^{2}$; IQR: -34.4 to -18.6 ) than those without AKI (median: $0.0 \mathrm{~mL} / \mathrm{min} / 1.73 \mathrm{~m}^{2}$; IQR: -9.6 to 0.0 ). AKI was diagnosed in all patients between $24-$ and 72-hour time frame after PBC administration: in two patients, AKI was diagnosed on the second day (24-48 hours) and in another two patients AKI was manifest on the third day (48-72 hours) after chemotherapy. Maximum creatinine was evident in all AKI patients at 72-hour time point. Oliguric AKI was recorded in none of the patients.

Eight patients $(25.0 \%)$ received carboplatin and 24 patients $(75.0 \%)$ cisplatin. No significant differences in age, BMI, cisplatin dose and cisplatin cycle as well as in baseline $\mathrm{sCr}$ and baseline eGFR between the groups "no AKI" and "AKI" were observed (Table 1). Primary disease was lymphoma in 13 patients (40.6\%) and solid tumors in 19 patients $(59.4 \%)$. The lymphoma group comprised 12 nonHodgkin lymphoma and one Hodgkin lymphoma. Among

Table I Baseline characteristics

\begin{tabular}{|c|c|c|c|c|c|c|c|c|}
\hline \multirow[t]{2}{*}{ Characteristics } & \multicolumn{3}{|l|}{ No AKI } & \multicolumn{3}{|l|}{ AKI } & \multirow[b]{2}{*}{$p$-value } & \multirow[t]{2}{*}{ Total, n (\%) } \\
\hline & n (\%) & Median & IQR & n (\%) & Median & IQR & & \\
\hline Total & $28(87.5)$ & & & $4(12.5)$ & & & & $32(100)$ \\
\hline Age (years) & & 62.5 & $51.5-73.3$ & & 55.5 & $49.0-69.3$ & 0.65 & \\
\hline Male gender & $18(64.3)$ & & & $4(100)$ & & & & $22(68.8)$ \\
\hline BMI $\left(\mathrm{kg} / \mathrm{m}^{2}\right)$ & & 24.8 & $22.0-28.5$ & & 27.7 & $23.8-31.4$ & 0.56 & \\
\hline Cisplatin & $20(83.3)$ & & & $4(16.7)$ & & & & $24(75.0)$ \\
\hline Dose $\left(\mathrm{mg} / \mathrm{m}^{2}\right)$ & & 73.5 & $30.0-100.0$ & & 100.0 & $81.3-100.0$ & 0.15 & \\
\hline Cycle & & 2.0 & $1.0-3.0$ & & 3.5 & $1.5-4.0$ & 0.29 & \\
\hline Carboplatin & $8(100)$ & & & $0(0)$ & & & & $8(25.0)$ \\
\hline Dose $\left(\mathrm{mg} / \mathrm{m}^{2}\right)$ & & 257.0 & $189.5-458.0$ & & NV & $N V$ & & \\
\hline Cycle & & 2.0 & $2.0-5.5$ & & NV & NV & & \\
\hline Baseline $\mathrm{s} C r(\mathrm{mg} / \mathrm{dL})$ & & 0.8 & $0.6-0.9$ & & 1.0 & $0.7-1.2$ & 0.48 & \\
\hline Baseline eGFR $\left(\mathrm{mL} / \mathrm{min} / 1.73 \mathrm{~m}^{2}\right)$ & & 94.1 & $85.5-103.7$ & & 81.9 & $62.0-104.8$ & 0.60 & \\
\hline Maximum sCr within 72 hours (mg/dL) & & 0.9 & $0.6-1.1$ & & 1.4 & I.I-2.0 & $0.0075^{*}$ & \\
\hline \multicolumn{9}{|l|}{ Minimum eGFR within 72 hours (mL/ } \\
\hline $\left.\min / 1.73 \mathrm{~m}^{2}\right)$ & & 90.3 & $77.6-99.0$ & & 50.7 & $37.2-79.5$ & $0.016^{*}$ & \\
\hline \multicolumn{9}{|l|}{$\mathrm{s} C r$ changes from baseline within } \\
\hline 72 hours $(\mathrm{mg} / \mathrm{dL})$ & & 0.0 & $0.0-0.1$ & & 0.5 & $0.3-0.8$ & $<0.000 I^{*}$ & \\
\hline \multicolumn{9}{|l|}{$\mathrm{s} C r$ changes from baseline within } \\
\hline 72 hours $(\%)$ & & 0.0 & $0.0-12.5$ & & 57.2 & $35.7-47.1$ & $<0.000 I^{*}$ & \\
\hline \multicolumn{9}{|l|}{ eGFR changes from baseline within } \\
\hline 72 hours $\left(\mathrm{mL} / \mathrm{min} / 1.73 \mathrm{~m}^{2}\right)$ & & 0.0 & -9.6 to 0.0 & & -28.2 & -34.4 to 18.6 & $0.0004 *$ & \\
\hline \multicolumn{9}{|l|}{ eGFR changes from baseline within } \\
\hline 72 hours $(\%)$ & & 0.0 & -15.4 to 0.0 & & -34.3 & -50.2 to 20.4 & $0.001 *$ & \\
\hline \multicolumn{9}{|l|}{ TIMP2 IGFBP7 prior to PBC ((ng/ } \\
\hline $\mathrm{mL})^{2} /(000)$ & & 0.12 & $0.04-0.20$ & & 0.35 & $0.27-0.89$ & $0.019 *$ & \\
\hline TIMP2 IGFBP7 after PBC $\left((\mathrm{ng} / \mathrm{mL})^{2} / / 000\right)$ & & 0.07 & $0.03-0.17$ & & 0.69 & $0.30-1.08$ & $0.04 *$ & \\
\hline \multicolumn{9}{|l|}{ Primary disease } \\
\hline Solid tumor & $17(89.5)$ & & & $2(10.5)$ & & & & $19(59.4)$ \\
\hline Lymphoma & II (84.6) & & & $2(I 5.4)$ & & & & $13(40.6)$ \\
\hline
\end{tabular}

Note: All subjects developed AKI stage I defined by KDIGO *p<0.05.

Abbreviations: AKI, acute kidney injury; BMI, body mass index; eGFR, estimated glomerular filtration rate; IQR, interquartile range; KDIGO, Kidney Disease: Improving Global Outcomes; NV, no value; PBC, platinum-based chemotherapy; sCr, serum creatinine. 
solid tumors, the individual malignancies were four testicular tumors, three bronchial carcinomas, two esophageal carcinomas, two breast carcinomas, two urothelial carcinomas, two ovarian cancers, one neuroendocrine carcinoma, one osteosarcoma, one peritoneal carcinoma and one extragonadal germ cell tumor.

\section{Biomarker results}

Urinary TIMP2-IGFBP7 values after the administration of PBC were significantly ( $p=0.04)$ higher in patients with AKI than those without AKI. The median urinary TIMP2-IGFBP7 level was 0.07 (IQR: 0.03-0.17) in the non-AKI group and 0.69 (IQR: 0.30-1.08) in the AKI group (Figure 2).

Similarly, urinary TIMP2-IGFBP7 values prior to the application of $\mathrm{PBC}$ differed significantly in patients developing AKI within 72 hours compared to patients without AKI $(p=0.019)$. The median value in the non-AKI group was 0.12 (IQR: 0.04-0.20), whereas in the AKI cohort the median urinary TIMP2·IGFBP7 level was 0.35 (IQR: 0.27-0.89) (Figure 3).

The TIMP2-IGFBP7 test characteristics for the prediction of AKI within 72 hours using the cutoff of 0.3 and urine samples after chemotherapy administration were as follows: sensitivity of 0.50 (95\% CI: $0.01-0.99)$, specificity of 0.87 (95\% CI: 0.66-0.97), NPV of 0.95 (95\% CI: 0.76-1.00) and PPV of 0.25 (95\% CI: $0.01-0.81)$. Most discriminatory cutoff was 0.28 with the sensitivity of 1.00 (95\% CI: $0.16-1.00)$ and specificity of 0.87 (95\% CI: $0.66-0.97)$. The AUC was 0.92 (95\% CI: 0.80-1.00) (Table 2), but in this analysis, there were only $n=2$ subjects in the AKI group.

Urinary TIMP2·IGFBP7 after PBC

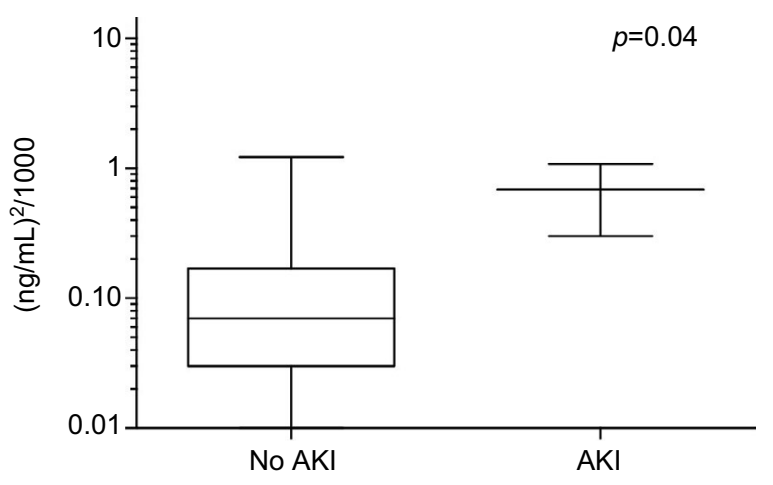

Figure 2 Urinary TIMP2 IGFBP7 after the administration of PBC in patients with malignant disease.

Notes: Patients developing AKI within 72 hours had significant higher urinary TIMP2 IGFBP7 values than patients without AKI ( $p=0.04)$. No AKI: median: 0.07 (IQR: 0.03-0.17). AKI: median: 0.69 (IQR: 0.30-I.08) ( $n=26)$. Box and whiskers show the IQR and total observed range, respectively. Horizontal line within the box shows the median.

Abbreviations: $\mathrm{AKI}$, acute kidney injury; IQR, interquartile range; $\mathrm{PBC}$, platinumbased chemotherapy.
Urinary TIMP2·IGFBP7 measured in samples prior to PBC application showed a sensitivity of 0.75 (95\% CI: 0.19-0.99), specificity of 0.82 (95\% CI: $0.63-0.94), \mathrm{NPV}$ of 0.96 (95\% CI: $0.79-1.00)$ and PPV of 0.38 (95\% CI: 0.09-0.76). Most discriminatory cutoff was 0.24 with the sensitivity of 1.00 (95\% CI: $0.40-1.00)$ and specificity of 0.82 (95\% CI: $0.63-0.94)$. The AUC was 0.86 (95\% CI: 0.73-0.98) (Table 3).

\section{Discussion}

Platinum-based chemotherapeutics are widely applied cytotoxic agents, but side effects are dose limiting and can lead to serious complications. ${ }^{6,7}$ The most important side effect is nephrotoxicity, which often represents a doseand therapy-restricting factor. ${ }^{21}$ Acute kidney impairment appears approximately in one-third of the patients receiving cisplatin chemotherapy. ${ }^{6,7}$ For the prediction of AKI risk or early detection, $\mathrm{sCr}$ is not sufficient because of a delayed increase and a creatinine-blind range. ${ }^{32}$ Often tubular damage is evident without a notable rise in $\mathrm{sCr} .{ }^{33}$ Acute Dialysis Quality Initiative Consensus Conference has proposed the inclusion of tubular damage markers for the definition of AKI. ${ }^{22}$ Urinary TIMP2-IGFBP7 seems to be one of the most promising tubular damage biomarkers for the early detection of AKI. ${ }^{23}$

In this study, significantly $(p=0.04)$ higher median urinary TIMP2-IGFBP7 levels, collected from the patients after receiving $\mathrm{PBC}$, were observed in the AKI group (Figure 2). Interestingly, a significant $(p=0.019)$ increase in urinary TIMP2 $\cdot$ IGFBP7 prior to $\mathrm{PBC}$ administration was evident in patients developing AKI within 72 hours after chemotherapy application (Figure 3 ). This could imply that those patients developing AKI already had tubular stress, and therefore had probably an increased vulnerability for the toxic effects of the PBC agents.

Both findings suggest that urinary TIMP $2 \cdot$ IGFBP 7 could be a helpful tool for the identification of patients who are at risk for developing $\mathrm{AKI}$. As a consequence, for patients with urinary TIMP $2 \cdot$ IGFBP7 levels $>0.3$, intensified prevention strategies could be implemented for the reduction in AKI rates after $\mathrm{PBC}$ application. Conversely, maybe higher PBC doses could be tolerated by the kidneys when urinary TIMP2·IGFBP7 levels are low.

Previous works described increased biomarker concentrations such as KIM-1, calbindin and TIMP-1 in cisplatin toxicity models in vitro and in vivo, confirming the relevance of urinary biomarkers in cisplatin nephrotoxicity. ${ }^{34}$ Our findings are comparable to preceding studies examining 
Urinary TIMP2·IGFBP7 prior to PBC

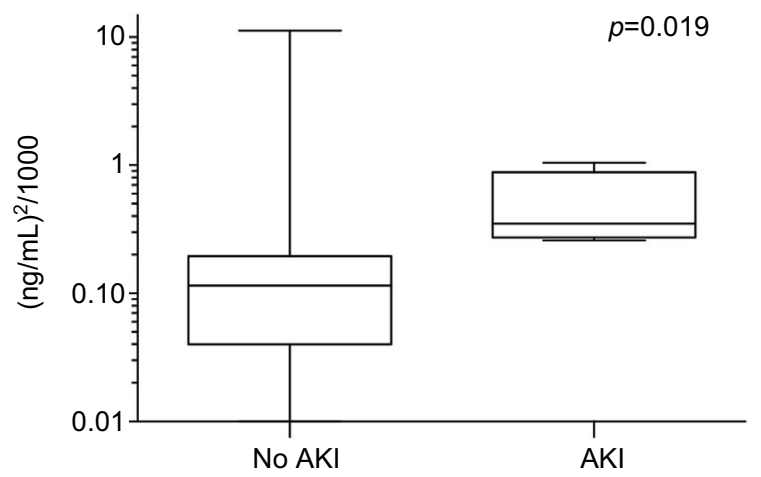

Figure 3 Urinary TIMP2 IGFBP7 prior to the administration of PBC in patients with malignant disease.

Notes: Patients developing AKI within 72 hours had significant higher urinary TIMP2 IGFBP7 values than patients without AKI $(p=0.019)$. No AKI: median: 0.12 (IQR: 0.04-0.20). AKI: median: 0.35 (IQR: 0.27-0.89) $(n=32)$. Box and whiskers show the IQR and total observed range, respectively. Horizontal line within the box shows the median.

Abbreviations: AKI, acute kidney injury; IQR, interquartile range; PBC, platinumbased chemotherapy.

AKI biomarkers in patients receiving cisplatin: Lin et al ${ }^{35}$ demonstrated an AUC of 0.865 for NGAL levels and AKI development. Comparable AUCs were described by Tekce et $\mathrm{a}^{36}$ for urinary KIM-1 levels after PBC application. In our study, urinary TIMP2-IGFBP7 had an AUC of 0.92 $(0.80-1.03)$ when using the urinary sample after PBC application. Surprisingly, we also showed that urinary biomarkers collected prior to $\mathrm{PBC}$ application can also predict $\mathrm{AKI}$ risk: the AUC of TIMP2-IGFBP7 for samples collected before $\mathrm{PBC}$ was $0.86(0.73-0.98)$.

We consecutively enrolled all patients getting PBC; for that reason, we also included patients receiving carboplatin. Carboplatin is known to have less serious, but still existing nephrotoxic effects. ${ }^{20}$ Previously, increased damage biomarker levels, e.g., for NGAL, were also shown in patients receiving carboplatin chemotherapy. ${ }^{37}$ Interestingly, in our study, all patients who manifested AKI within 72 hours received cisplatin.

In this study, we analyzed the performance of TIMP2-IGFBP7 at the cutoff of 0.3 , but we could not analyze the higher 2.0 cutoff because of insufficient sample size. Further studies with larger sample sizes are needed to confirm our findings and to analyze the performance of urinary TIMP2-IGFBP7 at the cutoff of 2.0 in PBC-receiving patients. In this cohort, the most discriminatory cutoff was 0.28 for samples gathered after PBC administration or 0.24 in samples collected prior to chemotherapy. Both cutoffs were close to the validated and established cutoff of 0.3 , implicating that the usage of 0.3 cutoff seems to be appropriate in this setting.

According to our data, cell cycle arrest biomarkers such as TIMP2 and IGFBP7 seem to play an important role in AKI due to PBC therapy. Cell cycle abnormalities are already described in several renal diseases as well in AKI. ${ }^{38,39}$ Previous studies showed that quiescent renal tubular cells reenter the cell cycle during AKI. ${ }^{40-42}$ Similarly, one pathophysiologic mechanism in cisplatin nephrotoxicity is dysregulation of cell cycle proteins. ${ }^{41} \mathrm{Yu}$ et $\mathrm{al}^{43}$ demonstrated that cell cycle inhibition can prevent renal proximal tubule cells from cisplatin-induced cell death. Therefore, TIMP2 and IGFBP7 molecules, known to be involved in G2 cell cycle arrest, ${ }^{23}$ could serve as a potential target for cell cycle inhibition and could reduce the incidence of PBC-related AKI. Under inhibition, maybe higher chemotherapy doses could be achieved, improving effectiveness of the therapy.

Our main limitation is the small sample size - the results provide a first clue for the usefulness of urinary TIMP2-IGFBP7, but further and larger studies are needed to confirm our findings.

Table 2 Urinary TIMP2 IGFBP7 sample after chemotherapy administration using the cutoff of 0.3

\begin{tabular}{lllll}
\hline Sensitivity & Specificity & NPV & PPV & AUC \\
\hline $0.50(0.01-0.99)$ & $0.87(0.66-0.97)$ & $0.95(0.76-1.00)$ & $0.25(0.01-0.81)$ & $0.92(0.80-1.00)$ \\
\hline
\end{tabular}

Note: Operating characteristics $(95 \% \mathrm{Cl})$ for the prediction of AKI within 72 hours after chemotherapy administration in patients receiving PBC ( $\mathrm{n}=26)$.

Abbreviations: AKI, acute kidney injury; AUC, area under the ROC curve; $\mathrm{Cl}$, confidence interval; NPV, negative predictive value; PBC, platinum-based chemotherapy; PPV, positive predictive value; ROC, receiver operating characteristic.

Table 3 Urinary TIMP2 IGFBP7 sample prior to chemotherapy administration using the cutoff of 0.3

\begin{tabular}{lllll}
\hline Sensitivity & Specificity & NPV & PPV & AUC \\
\hline $0.75(0.19-0.99)$ & $0.82(0.63-0.94)$ & $0.96(0.79-1.00)$ & $0.38(0.09-0.76)$ & $0.86(0.73-0.98)$ \\
\hline
\end{tabular}

Note: Operating characteristics $(95 \% \mathrm{Cl})$ for the prediction of $\mathrm{AKI}$ within 72 hours after chemotherapy administration in patients receiving PBC ( $\mathrm{n}=32)$.

Abbreviations: $\mathrm{AKI}$, acute kidney injury; $\mathrm{AUC}$, area under the ROC curve; $\mathrm{Cl}$, confidence interval; NPV, negative predictive value; PBC, platinum-based chemotherapy; PPV, positive predictive value; ROC, receive operating characteristic. 


\section{Conclusion}

Urinary TIMP2-IGFBP7 measured in specimens gathered after PBC application may be a useful tool to identify patients who are at risk for developing platinum-induced AKI. Surprisingly, urinary TIMP2-IGFBP7 measured in samples collected prior to PBC administration could also serve as an indicator of AKI risk and may reflect the individual vulnerability to PBC toxicity. Therefore, TIMP2 and IGFBP7 could be used as a potential target for cell cycle inhibition to prevent the incidence of platinum-induced AKI in future, but larger studies are needed to confirm our results.

\section{Abbreviations}

ADQI, Acute Dialysis Quality Initiative; AKI, acute kidney injury; AUC, area under the ROC curves; BMI, body mass index; CI, confidence interval; CKD-EPI, Chronic Kidney Disease Epidemiology Collaboration; FDA, Food and Drug Administration; eGFR, estimated GFR; GFR, glomerular filtration rate; IGFBP7, insulin-like growth factor-binding protein-7; IQR, interquartile range; KDIGO, Kidney Disease: Improving Global Outcomes; NGAL, neutrophil gelatinase-associated lipocalin; NPV, negative predictive value; $\mathrm{PBC}$, platinum-based chemotherapy; PPV, positive predictive value; ROC, receiver operating characteristic; sCr, serum creatinine; TIMP-2, tissue inhibitor of metalloproteinases- 2 .

\section{Acknowledgments}

This study was supported by the Robert-Bosch Foundation (Stuttgart, Germany). We thank our study nurses B Rettenmaier and A Schwab for their support. All data underlying the findings are within the paper or available upon reasonable request from the corresponding author.

\section{Disclosure}

MK received lecture honoraria from Astute Medical. JS has received consulting fees from Astute Medical. The other authors report no conflicts of interest in this work.

\section{References}

1. Rosenberg B, Vancamp L, Krigas T. Inhibition of cell division in Escherichia coli by electrolysis products from a platinum electrode. Nature. 1965;205:698-699.

2. Rosenberg B, VanCamp L, Trosko JE, Mansour VH. Platinum compounds: a new class of potent antitumour agents. Nature. 1969;222(5191):385-386.

3. Murphy BA, Gerber DE, Schiller JH, et al. Chemotherapy and molecular targeted therapy of human cancer. In: Skeel RT, Khleif SN, editors. Handbook of Cancer Chemotherapy. Philadelphia: Lippincott Williams \& Wilkins; 2011:69-523.
4. Curran WJ. New chemotherapeutic agents: update of major chemoradiation trials in solid tumors. Oncology. 2002;63(suppl 2):S29-S38.

5. Chu G, Mantin R, Shen YM, Baskett G, Sussman H. Massive cisplatin overdose by accidental substitution for carboplatin. Toxicity and management. Cancer. 1993;72(12):3707-3714.

6. Lebwohl D, Canetta R. Clinical development of platinum complexes in cancer therapy: an historical perspective and an update. Eur J Cancer. 1998;34(10):1522-1534.

7. Ries F, Klastersky J. Nephrotoxicity induced by cancer chemotherapy with special emphasis on cisplatin toxicity. Am J Kidney Dis. 1986;8(5):368-379.

8. Chertow GM, Levy EM, Hammermeister KE, et al. Independent association between acute renal failure and mortality following cardiac surgery. Am J Med. 1998;104(4):343-348

9. Ramesh G, Reeves WB. Inflammatory cytokines in acute renal failure. Kidney Int. 2004;66(suppl 91):S56-S61.

10. Deng J, Kohda Y, Chiao H, et al. Interleukin-10 inhibits ischemic and cisplatin-induced acute renal injury. Kidney Int. 2001;60(6):2118-2128.

11. Ramesh G, Reeves WB. Salicylate reduces cisplatin nephrotoxicity by inhibition of tumor necrosis factor-alpha. Kidney Int. 2004;65(2):490-499.

12. Luke DR, Vadiei K, Lopez-Berestein G. Role of vascular congestion in cisplatin-induced acute renal failure in the rat. Nephrol Dial Transplant. 1992;7(1):1-7.

13. Winston JA, Safirstein R. Reduced renal blood flow in early cisplatininduced acute renal failure in the rat. Am JPhysiol. 1985;249(4):F490-F496.

14. Kawai Y, Nakao T, Kunimura N, Kohda Y, Gemba M. Relationship of intracellular calcium and oxygen radicals to Cisplatin-related renal cell injury. J Pharmacol Sci. 2006;100(1):65-72.

15. Gonzales-Vitale JC, Hayes DM, Cvitkovic E, Sternberg SS. The renal pathology in clinical trials of cis-platinum (II) diamminedichloride. Cancer. 1977;39(4):1362-1371.

16. Dobyan DC, Levi J, Jacobs C, Kosek J, Weiner MW. Mechanism of cisplatinum nephrotoxicity: II. Morphologic observations. J Pharmacol Exp Ther. 1980;213(3):551-556.

17. Cornelison TL, Reed E. Nephrotoxicity and hydration management for cisplatin, carboplatin, and ormaplatin. Gynecol Oncol. 1993;50(2):147-158.

18. Bajorin DF, Bosl GJ, Alcock NW, Niedzwiecki D, Gallina E, Shurgot B. Pharmacokinetics of cis-diamminedichloroplatinum(II) after administration in hypertonic saline. Cancer Res. 1986;46(11):5969-5972.

19. Pasetto LM, D'Andrea MR, Brandes AA, et al. The development of platinum compounds and their possible combination. Crit Rev Oncol Hematol. 2006;60(1):59-75.

20. English MW, Skinner R, Pearson AD, Price L, Wyllie R, Craft AW. Dose-related nephrotoxicity of carboplatin in children. $\mathrm{Br} J$ Cancer. 1999;81(2):336-341.

21. Hoek J, Bloemendal KM, van der Velden LA, et al. Nephrotoxicity as a dose-limiting factor in a high-dose cisplatin-based chemoradiotherapy regimen for head and neck carcinomas. Cancers (Basel). 2016;8(2):E21.

22. Murray PT, Mehta RL, Shaw A, et al. Potential use of biomarkers in acute kidney injury: report and summary of recommendations from the 10th Acute Dialysis Quality Initiative consensus conference. Kidney Int. 2014;85(3):513-521.

23. Kashani K, Al-Khafaji A, Ardiles T, et al. Discovery and validation of cell cycle arrest biomarkers in human acute kidney injury. Crit Care. 2013;17(1):R25.

24. Bihorac A, Chawla LS, Shaw AD, et al. Validation of cell-cycle arrest biomarkers for acute kidney injury using clinical adjudication. $\mathrm{Am} J$ Respir Crit Care Med. 2014;189(8):932-939.

25. Meersch M, Schmidt C, Van Aken H, et al. Validation of cell-cycle arrest biomarkers for acute kidney injury after pediatric cardiac surgery. PLoS One. 2014;9(10):e110865.

26. Hoste EA, McCullough PA, Kashani K, et al. Derivation and validation of cutoffs for clinical use of cell cycle arrest biomarkers. Nephrol Dial Transplant. 2014;29(11):2054-2061. 
27. Kimmel M, Shi J, Latus J, et al. Association of renal stress/damage and filtration biomarkers with subsequent AKI during hospitalization among patients presenting to the emergency department. Clin JAm Soc Nephrol. 2016;11(6):938-946.

28. Kimmel M, Shi J, Wasser C, Biegger D, Alscher MD, Schanz MB. Urinary TIMP2-IGFBP7 - novel biomarkers to predict acute kidney injury. Am J Nephrol. 2016;43(5):375-382.

29. FDA [webpage on the Internet]. FDA News Release: FDA Allows Marketing of the First Test to Assess Risk of Developing Acute Kidney Injury; 2014. Available from: https://wayback.archive-it. org/7993/20170112222827/http://www.fda.gov/NewsEvents/Newsroom/PressAnnouncements/ucm412910.htm. Accessed April 27, 2017.

30. Kidney Disease: Improving Global Outcomes (KDIGO) Acute Kidney Injury Work Group. KDIGO clinical practice guideline for acute kidney injury. Kidney Int Suppl. 2012;2(1):1-138.

31. Levey AS, Stevens LA, Schmid CH, et al. A new equation to estimate glomerular filtration rate. Ann Intern Med. 2009;150(9):604-612.

32. Dennen P, Parikh CR. Biomarkers of acute kidney injury: can we replace serum creatinine? Clin Nephrol. 2007;68(5):269-278.

33. Haase M, Kellum JA, Ronco C. Subclinical AKI - an emerging syndrome with important consequences. Nat Rev Nephrol. 2012;8(12):735-739.

34. Sohn SJ, Kim SY, Kim HS, et al. In vitro evaluation of biomarkers for cisplatin-induced nephrotoxicity using HK-2 human kidney epithelial cells. Toxicol Lett. 2013;217(3):235-242.
35. Lin HY, Lee SC, Lin SF, et al. Urinary neutrophil gelatinase-associated lipocalin levels predict cisplatin-induced acute kidney injury better than albuminuria or urinary cystatin C levels. Kaohsiung J Med Sci. 2013;29(6):304-311.

36. Tekce BK, Uyeturk U, Tekce H, Uyeturk U, Aktas G, Akkaya A. Does the kidney injury molecule-1 predict cisplatin-induced kidney injury in early stage? Ann Clin Biochem. 2015;52(pt 1):88-94.

37. Seker MM, Deveci K, Seker A, et al. Predictive role of neutrophil gelatinase-associated lipocalin in early diagnosis of platin-induced renal injury. Asian Pac J Cancer Prev. 2015;16(2):407-410.

38. Shankland SJ. Cell-cycle control and renal disease. Kidney Int. 1997;52(2):294-308.

39. Price PM, Safirstein RL, Megyesi J. The cell cycle and acute kidney injury. Kidney Int. 2009;76(6):604-613.

40. Price PM, Megyesi J, Saf Irstein RL. Cell cycle regulation: repair and regeneration in acute renal failure. Kidney Int. 2004;66(2):509-514.

41. Megyesi J, Safirstein RL, Price PM. Induction of p21WAF1/CIP1/SDI in kidney tubule cells affects the course of cisplatin-induced acute renal failure. J Clin Invest. 1998;101(4):777-782.

42. Megyesi J, Andrade L, Vieira JM Jr, Safirstein RL, Price PM. Coordination of the cell cycle is an important determinant of the syndrome of acute renal failure. Am J Physiol Renal Physiol. 2002;283(4):F810-F816.

43. Yu F, Megyesi J, Price PM. Cytoplasmic initiation of cisplatin cytotoxicity. Am J Physiol Renal Physiol. 2008;295(1):F44-F52.

\section{Publish your work in this journal}

The International Journal of Nephrology and Renovascular Disease is an international, peer-reviewed open access journal focusing on the pathophysiology of the kidney and vascular supply. Epidemiology, screening, diagnosis, and treatment interventions are covered as well as basic science, biochemical and immunological studies. The manuscript management system is completely online and includes a very quick and fair peer-review system, which is all easy to use. Visit http://www. dovepress.com/testimonials.php to read real quotes from published authors. 